\title{
A MULTIVARIATE GENERALIZATION OF THE GENERALIZED POISSON DISTRIBUTION
}

\author{
BY \\ RALUCA VeRnic \\ "Ovidius" University of Constanta, Romania
}

\begin{abstract}
This paper proposes a multivariate generalization of the generalized Poisson distribution. Its definition and main properties are given. The parameters are estimated by the method of moments.
\end{abstract}

\section{KEYWORDS}

Multivariate generalized Poisson distribution $\left(\mathrm{MGP}_{m}\right)$; generalized Poisson distribution (GPD); bivariate generalized Poisson distribution (BGPD).

\section{INTRODUCTION}

The univariate generalized Poisson distribution (GPD), introduced by CoNsUL and JAIN (1973), is a well-studied alternative to the standard Poisson distribution. CONSUL (1989) provided a guide to the current state of modeling with the GPD at that time, and documented many real life examples. GPD has also been making appearances in the actuarial literature (see GERBER, 1990; GoovaerTs and KaAs, 1991; Kling and GoovaerTs, 1993; AmbagaSPITIYA and BALAKRISHNAN, 1994 etc.). A bivariate generalization was developed by VERNIC (1997) and was applied in the insurance field.

The multivariate generalization that we present in this paper is derived from the GPD in a similar way with the BGPD. In consequence, the BGPD can be obtained from the $\mathrm{MGP}_{m}$ for $m=2$. In section 2 we present some properties of the $\mathrm{MGP}_{m}$. The method of moments is used in section 3 for the estimation of the parameters. In section 4 the particular case of the BGPD is considered together with its application in the insurance field, based on the paper of VERNIC (1997) and illustrated with a numerical example. Since the BGPD is well fitted to the aggregate amount of claims for a compound class of policies submitted to claims of two kinds whose yearly frequencies are a priori dependent, it is natural to consider that the $\mathrm{MGP}_{m}$ is a good candidate for the aggregate amount of claims for a class of policies submitted to claims of $m$ kinds. 


\section{The MUltivariate Generalized POISSON DisTRibution}

\subsection{Development of the distribution}

If $N \sim G P D(\lambda, \theta)$, then its probability function (p.f.) is given by (CoNSUL and SHOUKRI, 1985)

$f(n)=P(N=n)=\left\{\begin{array}{l}\frac{1}{n !} \lambda(\lambda+n \theta)^{n-1} \exp \{-\lambda-n \theta\}, \quad n=0,1, \ldots \\ 0, \quad \text { for } n>q \text { when } \theta<0\end{array}\right.$

and zero otherwise, where $\lambda>0, \max (-1,-\lambda / q) \leq \theta<1$ and $q \geq 4$ is the largest positive integer for which $\lambda+\theta q>0$ when $\theta<0$.

VERNIC (1997) used the trivariate reduction method to construct the BGPD in the following way: let $N_{i}, i=1,2,3$, be independent generalized Poisson random variables (r.v.), $N_{i} \sim G P D\left(\lambda_{i}, \theta_{i}\right), i=1,2,3$, and let $X=N_{1}+N_{3}$ and $Y=N_{2}+N_{3}$. Then $(X, Y) \sim B G P D\left(\lambda_{i}, \theta_{i} ; i=1,2,3\right)$.

Similarly, we obtain the $m$-dimensional generalized Poisson distribution by taking $(m+1)$ independent generalized Poisson random variables, $N_{i} \sim G P D\left(\lambda_{i}, \theta_{i}\right), i=0, \ldots, m$, and considering $X_{1}=N_{1}+N_{0}, \ldots, X_{m}=$ $N_{m}+N_{0}$. Then $\left(X_{1}, \ldots, X_{m}\right) \sim M G P_{m}(\Lambda, \Theta)$, where $\Lambda=\left(\lambda_{0}, \lambda_{1}, \ldots, \lambda_{m}\right)$ and $\Theta=\left(\theta_{0}, \theta_{1}, \ldots, \theta_{m}\right)$. This method can be called the multivariate reduction method, as an extension of the trivariate reduction method.

It is easy to see that the joint p.f. of $\left(X_{1}, \ldots, X_{m}\right)$ reads

$$
\begin{aligned}
p\left(x_{1}, \ldots, x_{m}\right) & =P\left(X_{1}=x_{1}, \ldots, X_{m}=x_{m}\right)= \\
& =\sum_{k=0}^{\min \left\{x_{1}, \ldots, x_{m}\right\}} f_{1}\left(x_{1}-k\right) \cdot \ldots \cdot f_{m}\left(x_{m}-k\right) f_{0}(k),
\end{aligned}
$$

where $f_{i}$ is the p.f. of the r.v. $N_{i}$.

Using (2.1) in (2.2) we get

$$
\begin{aligned}
& p\left(x_{1}, \ldots, x_{m}\right)=\left(\prod_{j=0}^{m} \lambda_{j}\right) \exp \left\{-\lambda-\sum_{j=1}^{m} x_{j} \theta_{j}\right\} . \\
& \cdot \sum_{k=0}^{\min \left\{x_{1}, \ldots, x_{m}\right\}}\left(\prod_{j=1}^{m} \frac{\left[\lambda_{j}+\left(x_{j}-k\right) \theta_{j}\right]^{x_{j}-k-1}}{\left(x_{j}-k\right) !}\right) . \\
& \cdot \frac{\left(\lambda_{0}+k \theta_{0}\right)^{k-1}}{k !} \exp \left\{k\left(\sum_{j=1}^{m} \theta_{j}-\theta_{0}\right)\right\} \\
& x_{1}, \ldots, x_{m}=0,1,2, \ldots
\end{aligned}
$$

where $\lambda=\sum_{j=0}^{m} \lambda_{j}$ and $0 !=1$. 


\subsection{Properties of the distribution}

We will first make some remarks on the GPD.

The GPD reduces to the Poisson distribution when $\theta=0$ and it possesses the twin properties of over-dispersion and under-dispersion according as $\theta>0$ or $\theta<0$. When $\theta$ is negative, the GPD model includes a truncation due to the fact that $f(n)=0$ for all $n>q$ (see 2.1). In the following, the moments expressions and the other formulas for the GPD are valid only for the case $\lambda>0,0 \leq \theta<1$ and $q=\infty$, as discussed in SCOLLNIK (1998). This is a point frequently misrepresented in the literature.

In conclusion, we will assume for simplicity that $\theta>0$. From AMBGASPITIYA and BALAKRISHNAN (1994) we have the following formulas for $N \sim G P D(\lambda, \theta)$ :

- the probability generating function (p.g.f.)

$$
\Pi_{N}(t)=\exp \left\{-\frac{\lambda}{\theta}[W(-\theta t \exp \{-\theta\})+\theta]\right\} .
$$

- the moment generating function (m.g.f.)

$$
M_{N}(t)=\exp \left\{-\frac{\lambda}{\theta}[W(-\theta \exp \{-\theta+t\})+\theta]\right\},
$$

where the Lambert $W$ function is defined as $W(x) \exp \{W(x)\}=x$. For more details about this function see CoRLESS et al. (1996).

- the first four central moments

$$
\left\{\begin{array}{l}
E(N)=\mu_{1}=\lambda M ; \quad \operatorname{Var}(N)=\mu_{2}=\lambda M^{3} \\
\mu_{3}=\lambda(3 M-2) M^{4} ; \quad \mu_{4}=3 \lambda^{2} M^{6}+\lambda\left(15 M^{2}-20 M+6\right) M^{5}
\end{array}\right\},
$$

where $M=(1-\theta)^{-1}$.

The probability generating function of the $\mathrm{MGP}_{\boldsymbol{m}}$

Let now $\Pi_{i}(t)$ denote the p.g.f. of the r.v. $N_{i}, i=0, \ldots, m$. Then the joint p.g.f. of $\left(X_{1}, \ldots, X_{m}\right)$ is

$$
\begin{aligned}
\Pi\left(t_{1}, \ldots, t_{m}\right) & =E\left(t_{1}^{X_{1}} \cdot \ldots \cdot t_{m}^{X_{m}}\right)=E\left(t_{1}^{N_{1}} \cdot \ldots \cdot t_{m}^{N_{m}}\left(t_{1} \cdot \ldots \cdot t_{m}\right)^{N_{0}}\right)= \\
& =\Pi_{1}\left(t_{1}\right) \cdot \ldots \cdot \Pi_{m}\left(t_{m}\right) \Pi_{0}\left(t_{1} \cdot \ldots \cdot t_{m}\right) .
\end{aligned}
$$

Using (2.4) in (2.7) and assuming that $\theta_{i}>0, i=0, \ldots, m$, we have

$$
\Pi\left(t_{1}, \ldots, t_{m}\right)=\exp \left\{-\sum_{i=1}^{m} \frac{\lambda_{i}}{\theta_{i}} W\left(-\theta_{i} t_{i} e^{-\theta_{i}}\right)-\frac{\lambda_{0}}{\theta_{0}} W\left(-\theta_{0} e^{-\theta_{0}} \prod_{i=1}^{m} t_{i}\right)-\lambda\right\} .
$$


The moment generating function of the $\mathrm{MGP}_{\boldsymbol{m}}$

If the m.g.f. of $N_{i}$ is $M_{i}(t), i=0, \ldots, m$, then the m.g.f. of $\left(X_{1}, \ldots, X_{m}\right)$ is

$$
\begin{aligned}
M\left(t_{1}, \ldots, t_{m}\right) & =E\left(\exp \left\{t_{1} X_{1}+\ldots+t_{m} X_{m}\right\}\right)=E\left(e^{t_{1} N_{1}} \cdot \ldots \cdot e^{t_{m} N_{m}} e^{\left(t_{1}+\ldots+t_{m}\right) N_{0}}\right) \\
& =M_{1}\left(t_{1}\right) \cdot \ldots \cdot M_{m}\left(t_{m}\right) M_{0}\left(t_{1}+\ldots+t_{m}\right) .
\end{aligned}
$$

Using (2.5) in (2.8), the joint m.g.f. is given for $\theta_{i}>0, i=0, \ldots, m$, by

$$
\begin{aligned}
& M\left(t_{1}, \ldots, t_{m}\right)= \\
& \exp \left\{-\sum_{i=1}^{m} \frac{\lambda_{i}}{\theta_{i}} W\left(-\theta_{i} \exp \left\{-\theta_{i}+t_{i}\right\}\right)-\frac{\lambda_{0}}{\theta_{0}} W\left(-\theta_{0} \exp \left\{-\theta_{0}+\sum_{i=1}^{m} t_{i}\right\}\right)-\lambda\right\} .
\end{aligned}
$$

Met $\mu_{r_{1}, \ldots, r_{m}}=E\left(\prod_{j=1}^{m}\left(X_{j}-E X_{j}\right)^{r_{j}}\right)$ be the $\left(r_{1}, \ldots, r_{m}\right)^{\text {th }}$ central moment of $\left(X_{1}, \ldots, X_{m}\right)$. The equation for $\mu_{r_{1}, \ldots, r_{m}}$ given $\mu_{k}^{(j)}$ the $k^{\text {th }}$ central moment of $N_{j}, j=0, \ldots, m$, results as follows

$$
\begin{aligned}
\mu_{r_{1}, \ldots, r_{m}} & =E\left[\prod_{j=1}^{m}\left(N_{j}-E N_{j}+N_{0}-E N_{0}\right)^{r_{j}}\right]= \\
& =E\left[\prod_{j=1}^{m} \sum_{i_{j}=0}^{r_{j}}\left(\begin{array}{l}
r_{j} \\
i_{j}
\end{array}\right)\left(N_{j}-E N_{j}\right)^{i_{j}}\left(N_{0}-E N_{0}\right)^{\left.r_{j}-i_{j}\right]}\right]= \\
& =\sum_{\left(i_{1}, \ldots, i_{m}\right)=(0, \ldots, 0)}^{\left(r_{1}, \ldots, r_{m}\right)}\left(\prod_{j=1}^{m}\left(\begin{array}{c}
r_{j} \\
i_{j}
\end{array}\right) \mu_{i_{j}}^{(j)}\right) \mu_{\sum_{j=1}^{m}\left(r_{j}-i_{j}\right)}^{(0)} .
\end{aligned}
$$

From (2.6) and the independence of $N_{j}, j=0, \ldots, m$, we also have for $\theta_{i}>0$, $i=0, \ldots, m$,

$$
\left\{\begin{array}{l}
E X_{i}=\lambda_{i} M_{i}+\lambda_{0} M_{0} \\
\operatorname{Var}\left(X_{i}\right)=\lambda_{i} M_{i}^{3}+\lambda_{0} M_{0}^{3}
\end{array}, \quad i=1, \ldots, m,\right.
$$

and from (2.9) we have, for example

$$
\left\{\begin{array}{l}
\mu_{110 \ldots 0}=\mu_{00.010 \ldots 010 \ldots 0}=\mu_{2}^{(0)}=\lambda_{0} M_{0}^{3} \\
\mu_{1110 \ldots 0}=\mu_{0 . .010 \ldots 010 \ldots 010 \ldots 0}=\mu_{3}^{(0)}=\lambda_{0}\left(3 M_{0}-2\right) M_{0}^{4} \\
\cdot \cdot \\
\cdot \\
\mu_{11 \ldots 1}=\mu_{m}^{(0)}
\end{array}\right.
$$




\section{Marginal distributions}

The marginal distributions are

$$
\begin{aligned}
& P\left(X_{i}=r\right)=P\left(N_{i}+N_{0}=r\right)=\lambda_{0} \lambda_{i} \exp \left\{-\left(\lambda_{0}+\lambda_{i}\right)-r \theta_{0}\right\} . \\
& \cdot \sum_{j=0}^{r} \frac{1}{j !(r-j) !}\left(\lambda_{i}+j \theta_{i}\right)^{j-1}\left(\lambda_{0}+(r-j) \theta_{0}\right)^{r-j-1} \exp \left\{-j\left(\theta_{i}-\theta_{0}\right)\right\}, \quad i=1, \ldots, m .
\end{aligned}
$$

In particular, if $\theta_{i}=\theta_{0}=\theta$, this reduces to $X_{i} \sim G P D\left(\lambda_{i}+\lambda_{0}, \theta\right)$. Elsewhere, $X_{i}$ is not a GPD.

Remark. From the development of the $\mathrm{MGP}_{m}$, it is easy to see that if $\left(X_{1}, \ldots, X_{m}\right) \sim M G P_{m}(\Lambda, \Theta)$, then for any $\left\{i_{1}, \ldots, i_{k}\right\} \subset\{1, \ldots, m\}$ with $2 \leq k<m,\left(X_{i_{1}}, \ldots, X_{i_{k}}\right) \sim M G P_{k}\left(\Lambda^{\prime}, \Theta^{\prime}\right)$, where $\Lambda^{\prime}=\left(\lambda_{0}, \lambda_{i_{1}}, \ldots, \lambda_{i_{k}}\right)$ and $\Theta^{\prime}=\left(\theta_{0}, \theta_{i_{1}}, \ldots, \theta_{i_{k}}\right)$.

For $k=1$ the remark is not always true. But if we consider the particular case $\theta_{0}=\theta_{1}=\ldots=\theta_{m}=\theta$, then from $\left(X_{1}, \ldots, X_{m}\right) \sim M G P_{m}(\Lambda, \Theta)$ it follows that $X_{i} \sim G P D\left(\lambda_{i}+\lambda_{0}, \theta\right), i=1, \ldots, m$.

\section{Recurrence relations}

The marginal p.f. can be computed using the univariate generalized Poisson distribution, as it is seen from

$$
\begin{aligned}
p(0, \ldots, 0)=\exp \{-\lambda\} & \\
p\left(0, \ldots, 0, x_{j}, 0, \ldots, 0\right) & =f_{j}\left(x_{j}\right)\left(\prod_{\substack{i=1 \\
i \neq j}}^{m} f_{i}(0)\right) f_{0}(0)= \\
& =f_{j}\left(x_{j}\right) \exp \left\{-\left(\lambda-\lambda_{j}\right)\right\}, j=1, \ldots, m, \quad x_{j}>0 .
\end{aligned}
$$

Given these probabilities, for $x_{j}>0, j=1, \ldots, m$, we have the following recurrence relation

$$
\begin{aligned}
p\left(x_{1}, \ldots, x_{m}\right)= & \lambda_{0} \exp \{(m-1) \lambda\} \sum_{k=0}^{\min \left\{x_{1}, \ldots, x_{m}\right\}}\left(\prod_{j=1}^{m} p\left(0, \ldots, 0, x_{j}-k, 0, \ldots, 0\right)\right) . \\
& \cdot \frac{\left(\lambda_{0}+k \theta_{0}\right)^{k-1}}{k !} \exp \left\{-k \theta_{0}\right\} .
\end{aligned}
$$




\section{ESTIMATION OF THE PARAMETERS: METHOD OF MOMENTS}

Let $\left(x_{1 i}, \ldots, x_{m i}\right), i=1, \ldots, n$ be a random sample of size $n$ from the population. We will assume that the frequency of the $m$-tuple $\left(s_{1}, \ldots, s_{m}\right)$ is $n_{s_{1}, \ldots, s_{m}}$ for $s_{1}, \ldots, s_{m}=0,1, \ldots$ We recall that $\sum_{s_{1}, \ldots, s_{m}} n_{s_{1}, \ldots, s_{m}}=n$. Also

$$
\left\{\begin{array}{l}
n_{+\ldots+s_{j}+\ldots+}=\sum_{\left\{s_{k} \mid k=1, \ldots, m, k \neq j\right\}} n_{s_{1}, \ldots, s_{m}} s_{1, \ldots, s_{m}} \\
n_{+\ldots+s_{i}+\ldots+s_{j}+\ldots+}=\sum_{\left\{s_{k} \mid k=1, \ldots, m, k \neq j, k \neq i\right\}} n_{s_{1}, \ldots, s_{m}}, \quad i<j
\end{array}\right.
$$

We denote

$$
\left\{\begin{array}{l}
\bar{x}_{j}=\frac{1}{n} \sum_{s_{j}} s_{j} n_{+\ldots+s_{j}+\ldots+} \\
\hat{\sigma}_{j}^{2}=\frac{1}{n} \sum_{s_{j}}\left(s_{j}-\bar{x}_{j}\right)^{2} n_{+} \ldots+s_{j}+\ldots+
\end{array}, \quad j=1, \ldots, m,\right.
$$

and, with the notations in (3.1)

$$
\left\{\begin{array}{l}
\overline{x_{i} x_{j}}=\frac{1}{n} \sum_{s_{i}, s_{j}} s_{i} s_{j} n_{+} \ldots+s_{i}+\ldots+s_{j}+\ldots+, \quad i<j \\
\overline{x_{i} x_{j} x_{k}}=\frac{1}{n} \sum_{s_{i}, s_{j}, s_{k}} s_{i} s_{j} s_{k} n_{+} \ldots+s_{i}+\ldots+s_{j}+\ldots+s_{k}+\ldots+, \quad i<j<k
\end{array}\right.
$$

It is easy to see that

$$
\left\{\begin{array}{rl}
\mu_{0 \ldots 010 \ldots 010 \ldots 0}=E\left(X_{i} X_{j}\right)-E\left(X_{i}\right) E\left(X_{j}\right), \quad i<j & \\
\mu_{0 \ldots 010 \ldots 010 \ldots 010 \ldots 0}=E\left(X_{i} X_{j} X_{k}\right)-E\left(X_{i} X_{j}\right) E\left(X_{k}\right)-E\left(X_{j} X_{k}\right) E\left(X_{i}\right)- \\
& -E\left(X_{i} X_{k}\right) E\left(X_{j}\right)+2 E\left(X_{i}\right) E\left(X_{j}\right) E\left(X_{k}\right), \quad i<j<k
\end{array},\right.
$$

so we can use the sample moments

$$
\left\{\begin{array}{c}
\hat{\mu}_{0 \ldots 010 \ldots 010 \ldots 0}=\overline{x_{i} x_{j}}-\bar{x}_{i} \bar{x}_{j}, \quad i<j \\
\hat{\mu}_{0 . \ldots 10 \ldots 010 \ldots 010 \ldots 0}=\overline{x_{i} x_{j} x_{k}}-\overline{x_{i} x_{j}} \bar{x}_{k}-\overline{x_{i} x_{k}} \bar{x}_{j}-\overline{x_{j} x_{k}} \bar{x}_{i}+ \\
+2 \bar{x}_{i} \bar{x}_{j} \bar{x}_{k}, \quad i<j<k
\end{array}\right.
$$




\section{The general method}

The classical method of moments consists of equating the sample moments to their populations equivalents, expressed in terms of the parameters. The number of moments required is equal to the number of parameters which equals $2(m+1)$. For example, using $(2.10),(2.11),(3.2)$ and (3.3), we can choose the following $2(m+1)$ equations

$$
\left\{\begin{array}{l}
\bar{x}_{j}=\lambda_{j} M_{j}+\lambda_{0} M_{0} \\
\hat{\sigma}_{j}^{2}=\lambda_{j} M_{j}^{3}+\lambda_{0} M_{0}^{3} \\
\hat{\mu}_{110 \ldots 0}=\lambda_{0} M_{0}^{3} \\
\hat{\mu}_{1110 \ldots 0}=\lambda_{0}\left(3 M_{0}-2\right) M_{0}^{4}
\end{array}, \quad j=1, \ldots, m .\right.
$$

Denoting $a=\frac{\hat{\mu}_{1110 \ldots 0}}{\hat{\mu}_{110 \ldots 0}}$, the solution of the system is

$$
\left\{\begin{array}{l}
M_{0}=\frac{1+\sqrt{1+3 a}}{3} \\
\lambda_{0}=\frac{\hat{\mu}_{110 \ldots 0}}{M_{0}^{3}} \\
M_{j}=\sqrt{\frac{\hat{\sigma}_{j}^{2}-\hat{\mu}_{110 \ldots 0}}{\bar{x}_{j}-\lambda_{0} M_{0}}}, \quad j=1, \ldots, m . \\
\lambda_{j}=\frac{\bar{x}_{j}-\lambda_{0} M_{0}}{M_{j}}
\end{array}\right.
$$

We used the fact that $\theta<1$, so $M=\frac{1}{1-\theta}>0$.

Particular case: $\theta_{0}=\theta_{1}=\ldots=\theta_{m}=\theta$, so $M_{0}=M_{1}=\ldots=M_{m}=M$.

Method I. The number of parameters is now $(m+2): \lambda_{0}, \ldots, \lambda_{m}$ and $M$, so we can use the following equations:

$$
\left\{\begin{array} { l } 
{ \overline { x } _ { j } = ( \lambda _ { j } + \lambda _ { 0 } ) M } \\
{ \hat { \mu } _ { 1 1 0 \ldots 0 } = \lambda _ { 0 } M ^ { 3 } } \\
{ \hat { \mu } _ { 1 1 1 0 . 0 } = \lambda _ { 0 } ( 3 M - 2 ) M ^ { 4 } }
\end{array} , \text { with the solution } \left\{\begin{array}{l}
M=\frac{1+\sqrt{1+3 a}}{3} \\
\lambda_{0}=\frac{\hat{\mu}_{110 \ldots 0}}{M^{3}} \\
\lambda_{j}=\frac{\bar{x}_{j}}{M}-\lambda_{0}
\end{array}, j=1, \ldots, m .\right.\right.
$$

Method II. Another possibility is to use the method of moments in combination with the zero cell frequency method. If we denote by $f_{0 \ldots 0}=\frac{n_{0 \ldots 0}}{n}$ the frequency of the cell $(0, \ldots, 0)$, we can consider the system

$$
\left\{\begin{array}{ll}
\text { I. } & f_{0 \ldots 0}=\exp \left\{-\left(\lambda_{0}+\ldots+\lambda_{m}\right)\right\} \\
\text { II. } & \bar{x}_{j}=\left(\lambda_{j}+\lambda_{0}\right) M \\
\text { III. } & \hat{\sigma}_{j}^{2}=\left(\lambda_{j}+\lambda_{0}\right) M^{3}
\end{array}, j=1, \ldots, m .\right.
$$


We have here $(2 m+1)$ equations. By summing equations $I$ and $I I$ separately, we get

$$
\left\{\begin{array}{l}
I V \cdot \sum_{j=1}^{m} \bar{x}_{j}=\left(\sum_{j=1}^{m} \lambda_{j}+m \lambda_{0}\right) M \\
V . \sum_{j=1}^{m} \hat{\sigma}_{j}^{2}=\left(\sum_{j=1}^{m} \lambda_{j}+m \lambda_{0}\right) M^{3}
\end{array}, \quad j=1, \ldots, m .\right.
$$

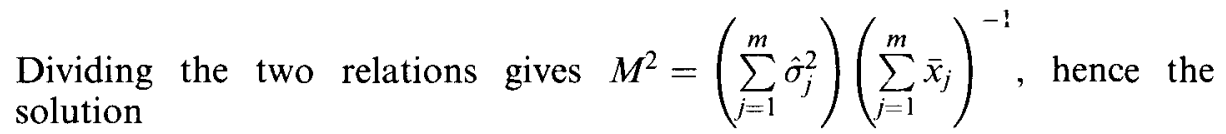

$$
M=\sqrt{\left(\sum_{j=1}^{m} \hat{\sigma}_{j}^{2}\right)\left(\sum_{j=1}^{m} \bar{x}_{j}\right)^{-1}} .
$$

From equation $I$ we have

$$
-\ln f_{0 \ldots 0}=\lambda_{0}+\sum_{j=1}^{m} \lambda_{j}
$$

and using equation $I V$ we are lead to

$$
-\ln f_{0 \ldots 0}=\lambda_{0}+\frac{1}{M} \sum_{j=1}^{m} \bar{x}_{j}-m \lambda_{0}
$$

so that

$$
\lambda_{0}=\frac{1}{m-1}\left(\frac{1}{M} \sum_{j=1}^{m} \bar{x}_{j}+\ln f_{0 \ldots 0}\right) .
$$

Then, from equation $I I$ we have

$$
\lambda_{j}=\frac{1}{M} \bar{x}_{j}-\lambda_{0}, j=1, \ldots, m
$$

Finally, the solution $\left(M, \lambda_{0}, \lambda_{j}, j=1, \ldots, m\right)$ is given by $(3.5),(3.6)$ and (3.7).

Remark. In method $I I$, the estimation of $M$ is based on the empirical moments from all $m$ variables, while in method $I$ only three variables are taken into consideration by $\hat{\mu}_{1110 \ldots 0}$. 


\section{PARTICULAR CASE: BIVARIATE GENERALIZED POISSON DISTRIBUTION (BGPD)}

Considering $m=2$, the multivariate generalized Poisson distribution reduces to the bivariate generalized Poisson distribution. The BGPD was introduced by VERNIC (1997) and was applied in the insurance field. The distribution was fitted to the aggregate amount of claims for a compound class of policies submitted to claims of two kinds whose yearly frequencies are a priori dependent. A comparative study with the classical bivariate Poisson distribution and with two bivariate mixed Poisson distributions has been carried out, based on two sets of data concerning natural events insurance in the U.S.A. and third party liability automobile insurance in France. The conclusion, after applying the $\chi^{2}$ goodness-of-fit test, is that the BGPD fits better to the data, so it can be considered as a valid alternative to the usual bivariate Poisson or mixed Poisson distributions. For more details see VERNIC (1997).

In the following, we will consider another example, based on the accident data of CRESSWELL and FROGATT (1963), with $X_{1}$ as the accidents in the first period and $X_{2}$ as the accidents in the second period. The data are given in table 1, first row in each cell.

The summary statistics for these data are:

$$
\begin{aligned}
& \bar{x}_{1}=1.0014, \quad \bar{x}_{2}=1.291, \quad \hat{\sigma}_{1}^{2}=1.1935, \quad \hat{\sigma}_{2}^{2}=1.5961, \\
& \hat{\mu}_{11}=0.3258, \quad \hat{\mu}_{21}=0.365 .
\end{aligned}
$$

Under the hypothesis $\left(X_{1}, X_{2}\right) \sim B G P D\left(\lambda_{0}, \lambda_{1}, \lambda_{2} ; \theta_{0}, \theta_{1}, \theta_{2}\right)$, we have from (3.4)

$$
\left\{\begin{array}{lll}
\theta_{0}=0.0286, & \theta_{1}=0.1057, & \theta_{2}=0.1200 \\
\lambda_{0}=0.2987, & \lambda_{1}=0.6206, & \lambda_{2}=0.8653
\end{array}\right\} .
$$

The theoretical frequencies in this case are given in table 1 , second row in each cell. After grouping in 32 categories: $(i, j)_{i=0.4 ; j=0.5} ;(0 . .4,6$ and above); ( 5 and above, 0 and above), we obtain $\chi_{o b s}^{2}=\sum(o b s-t h)^{2} / t h=25.935$ and a significance level ( $P$-value) verifying $0.45 \leq \hat{\alpha} \leq 0.75$. So the distribution is adequate.

We will now consider the particular case $\theta_{0}=\theta_{1}=\theta_{2}=\theta$, so that we have the hypothesis $\left(X_{1}, X_{2}\right) \sim B G P D\left(\lambda_{0}, \lambda_{1}, \lambda_{2} ; \theta\right)$. From (3.5), (3.6) and (3.7) we have $\theta=0.0935, \lambda_{0}=0.2778, \lambda_{1}=0.63, \lambda_{2}=0.8925$, and the theoretical frequencies are given in table 1 , last row in each cell. For the same categories we have $\chi_{o b s}^{2}=23.6082$ and $0.7 \leq \hat{\alpha} \leq 0.85$, so this particular distribution fits even better than the general one.

\section{ACKNOWLEDGEMENT}

The author is grateful to the referees for their helpful advice in revising the paper. 
TABLE 1

COMPARISON OF OBSERVED AND THEORETICAL FREQUENCIES

\begin{tabular}{|c|c|c|c|c|c|c|c|c|c|}
\hline $\begin{array}{l}X_{2} \\
X_{1}\end{array}$ & 0 & 1 & 2 & 3 & 4 & 5 & 6 & 7 & $\Sigma$ \\
\hline \multirow[t]{3}{*}{0} & 117 & 96 & 55 & 19 & 2 & 2 & 0 & 0 & 291 \\
\hline & 118.843 & 91.204 & 44.710 & 17.959 & 6.460 & 2.171 & 0.697 & 0.217 & 282.261 \\
\hline & 117 & 95.100 & 46.748 & 18.088 & 6.081 & 1.865 & 0.537 & 0.148 & 285.567 \\
\hline \multirow[t]{3}{*}{1} & 61 & 69 & 47 & 27 & 8 & 5 & 1 & 0 & 218 \\
\hline & 66.356 & 85.419 & 51.437 & 23.005 & 8.820 & 3.087 & 1.019 & 0.324 & 239.467 \\
\hline & 67.132 & 84.165 & 50.881 & 22,205 & 8.065 & 2.608 & 0.780 & 0.220 & 236.056 \\
\hline \multirow[t]{3}{*}{2} & 34 & 42 & 31 & 13 & 7 & 2 & 3 & 0 & 132 \\
\hline & 24.834 & 38.319 & 30.090 & 15.577 & 6.505 & 2.402 & 0.822 & 0.267 & 118.816 \\
\hline & 24.976 & 37.584 & 30.048 & 15.739 & 6.427 & 2.249 & 0.711 & 0.209 & 117.943 \\
\hline \multirow[t]{3}{*}{3} & 7 & 15 & 16 & 7 & 3 & 1 & 0 & 0 & 49 \\
\hline & 7.871 & 13.249 & 12.124 & 7.260 & 3.386 & 1.341 & 0.480 & 0.161 & 45.872 \\
\hline & 7.694 & 12.602 & 12.004 & 7.911 & 3.849 & 1.516 & 0.520 & 0.162 & 46.258 \\
\hline \multirow[t]{3}{*}{4} & 3 & 3 & 1 & 1 & 2 & 1 & 1 & 1 & 13 \\
\hline & 2.287 & 4.040 & 3.860 & 2.610 & 1.676 & 0.616 & 0.226 & 0.079 & 15.394 \\
\hline & 2.138 & 3.685 & 3.774 & 2.927 & 1.799 & 0.844 & 0.327 & 0.111 & 15.605 \\
\hline \multirow[t]{3}{*}{5} & 2 & 1 & 0 & 0 & 0 & 0 & 0 & 0 & 3 \\
\hline & 0.632 & 1.149 & 1.142 & 0.816 & 0.464 & 0.220 & 0.090 & 0.033 & 4.546 \\
\hline & 0.558 & 0.995 & 1.075 & 0.910 & 0.647 & 0.382 & 0.176 & 0.068 & 4.811 \\
\hline \multirow[t]{3}{*}{6} & 0 & 0 & 0 & 0 & 1 & 0 & 0 & 0 & 1 \\
\hline & 0.169 & 0.313 & 0.319 & 0.236 & 0.140 & 0.071 & 0.031 & 0.012 & 1.291 \\
\hline & 0.140 & 0.255 & 0.285 & 0.255 & 0.198 & 0.136 & 0.079 & 0.036 & 1.384 \\
\hline \multirow[t]{4}{*}{7} & 0 & 0 & 0 & 1 & 0 & 0 & 0 & 0 & 1 \\
\hline & 0.044 & 0.083 & 0.086 & 0.065 & 0.040 & 0.021 & 0.010 & 0.004 & 0.353 \\
\hline & 0.034 & 0.063 & 0.072 & 0.067 & 0.055 & 0.041 & 0.028 & 0.016 & 0.376 \\
\hline & 224 & 226 & 150 & 68 & 23 & 11 & 5 & 1 & \\
\hline \multirow[t]{2}{*}{$\Sigma$} & 221.036 & 233.776 & 143.768 & 67.528 & 27.491 & 9.929 & 3.375 & 1.097 & 708 \\
\hline & 219.672 & 234.449 & 144.887 & 68.102 & 27.121 & 9.641 & 3.158 & 0.970 & \\
\hline
\end{tabular}




\section{REFERENCES}

Ambagaspitiya, R.S. and Balakrishnan, N. (1994) On the compound generalized Poisson distributions. ASTIN Bulletin 24, 255-263.

Consul, P.C. (1989) Generalized Poisson Distributions: Properties and Applications. Marcel Dekker Inc., New York/Basel.

Consul, P.C. and JAIN, G.C. (1973) A generalization of the Poisson distribution. Technometrics 15, $791-799$.

Consul, P.C. and Shoukri, M.M. (1985) The generalized Poisson distribution when the sample mean is larger than the sample variance. Communications in Statistics - Simulation and Computation 14, 1533-1547.

Corless, R.M., Gonnet, G.H., Hare, D.E.G., JefFrey, D.J. and KNuth, D.E. (1996) On the Lambert W function. Advances in Computational Mathematics 5, 329-359.

Cresswell, W.L. and Froggatt, P. (1963) The causation of bus driver accidents. An epidemiological study. Oxford University Press, London.

GERBER, H.U. (1990) When does the surplus reach a given target? Insurance: Mathematics and Economics 9, 115-119.

GoovaerTs, M.J. and KaAs, R. (1991) Evaluating compound generalized Poisson distributions recursively. ASTIN Bulletin 21, 193-197.

KLING, B. and GoovaerTs, M. (1993) A note on compound generalized distributions. Scandinavian Actuarial Journal 1, 60-72.

SCOLLNIK, D.P.M. (1998) On the analysis of the truncated generalized Poisson distribution using a Bayesian method. ASTIN Bulletin 28, 135-152.

Vernic, R. (1997) On the bivariate generalized Poisson distribution. ASTIN Bulletin 27, 23-31.

RALUCA Vernic

Dept. of Mathematics and Informatics

"Ovidius" University of Constanta

124, Bd. Mamaia

8700 Constanta, Romania

E-mail: rvernic@univ-ovidius.ro 